\title{
Sistema de reconocimiento multimodal de emociones relacionadas al aprendizaje en dispositivos móviles
}

\author{
María Lucía Barrón-Estrada, Ramón Zatarain-Cabada, \\ Claudia Guadalupe Aispuro-Gallegos \\ Instituto Tecnológico de Culiacán, Culiacán, Sinaloa, \\ México \\ \{lbarron, rzatarain, m03171007\}@itculiacan.edu.mx
}

\begin{abstract}
Resumen. Gran variedad de sistemas reconocedores de emociones han sido implementados, pero pocos han logrado aplicarse en el mundo real debido al elevado costo de la tecnología necesaria y al bajo porcentaje de efectividad del reconocimiento, cuando no se trabaja con emociones espontáneas. Este artículo presenta la implementación de un sistema de reconocimiento multimodal de emociones usando dispositivos móviles y la creación de una base de datos afectiva por medio de una aplicación móvil. El reconocedor puede ser integrado fácilmente a una aplicación educativa móvil para identificar las emociones de un usuario mientras éste interactúa con el dispositivo. Las emociones que el sistema reconoce son compromiso y aburrimiento. La base de datos afectiva fue creada con emociones espontáneas de estudiantes que interactuaron con una aplicación móvil educativa llamada Duolingo y una aplicación móvil recolectora de información llamada EmoData. El sistema desarrollado tiene un porcentaje de efectividad aceptable y a medida que se incremente el número de registros de la base de datos afectiva, se espera que este porcentaje mejore.
\end{abstract}

Palabras clave: Computación afectiva móvil, reconocimiento de emociones, dispositivos móviles, reconocimiento voz, sensores de dispositivos móviles.

\section{Multimodal Recognition System of Learning Emotions in Mobile Devices}

\begin{abstract}
Many emotion recognizer systems have been developed, but only a few of them have been used in the real world. This may occur due to several reasons like: the high cost of required technology and the low rate of accuracy of recognition, when not working with spontaneous emotions. This paper presents the implementation of a multimodal emotion recognition system using mobile devices and the creation of an affective database populated by data collected from a mobile application. The recognizer can be easily integrated into a mobile educational application to identify user's emotions as they interact with the device. The recognized emotions are engagement and boredom. The affective database was created with spontaneous emotions of students who used a mobile educational application called Duolingo and a data gathering mobile application called EmoData. The developed system has an acceptable accuracy rate and by
\end{abstract}


increasing the amount of records of the affective database, it is expected this rate will be better.

Keywords: Mobile affective computing, emotion recognition, mobile devices, speech recognition, sensors of mobile devices.

\section{Introducción}

En los últimos años, el avance en el reconocimiento de emociones ha sido impresionante, generándose una gran variedad de sistemas reconocedores [1][2][3], bases de datos afectivas [4], técnicas de extracción de características y clasificación que logran mejorar ciertos aspectos como incrementar la precisión de reconocimiento y disminuir el tiempo de procesamiento, entre otros.

También se ha logrado confirmar la importancia del reconocimiento de emociones en el aprendizaje, definiéndolo como un componente clave en el desarrollo de aplicaciones, tutores y ambientes de aprendizaje inteligentes capaces de responder a las necesidades afectivas del estudiante. Cuando un estudiante está aprendiendo algo nuevo o aplicando sus conocimientos para resolver algún ejercicio pasa por varios estados emocionales, que pueden ser confusión, frustración, aburrimiento, compromiso u otros estados afectivos [5], [6], [7].

A pesar de estos avances, en esos sistemas persisten algunos problemas como: requieren de sensores o dispositivos invasivos y/o costosos, presentan dificultades asociadas con la recolección de datos de forma adecuada y realista para los modelos de aprendizaje máquina, poseen problemas pertenecientes a la generalización a través de contextos, tiempo y diferencias individuales [8], además existe una gran necesidad de recolectar emociones espontaneas a gran escala y una alternativa a este problema puede ser la captación a través de dispositivos móviles.

En la última década, el número de dispositivos móviles creció considerablemente al igual que las aplicaciones disponibles para ellos. El uso de las apps móviles aumentó un 58\% durante el 2015 y el tiempo que las personas dedican a los dispositivos móviles aumentó un $117 \%$ durante ese mismo año [9]. Además, creció el uso de todo tipo de apps (a excepción de los juegos, que tuvieron un retroceso del 1\%). Desde 2013, estos dispositivos ya había logrado conquistar a la sociedad pasando a formar parte de su vida diaria, las personas usan su teléfono inteligente en todo momento [3], el 40\% lo revisa cada 10 minutos, 3 de cada 4 personas revisan su teléfono inteligente al despertar, en periodos vacacionales 8 de cada 10 personas lo utiliza por largos periodos de tiempo y de forma muy frecuente.

Las personas pueden realizar numerosas actividades por medio de sus dispositivos móviles, debido a la gran cantidad de aplicaciones disponibles, sus capacidades de detección, procesamiento y comunicación y variedad de sus componentes: acelerómetro, giroscopio, sensor de luz, sensor de proximidad, cámara fotográfica, micrófono, entre otros.

Gran número de personas con diferentes características (edad, sexo, género, etc.) utilizan frecuentemente dispositivos móviles en cualquier lugar u hora el día, para realizar distintas actividades, esto genera grandes volúmenes de información que puede ser usada, entre otras cosas, para captar y reconocer emociones espontáneas de las personas en un ambiente natural. Por otro lado, la capacidad de procesamiento y 
componentes de estos dispositivos, puede llevar a cabo la tarea del reconocimiento en tiempo real en cualquier momento y lugar.

La principal contribución de este trabajo es la implementación de un sistema reconocedor de emociones usando dispositivos móviles, el cual puede ser integrado fácilmente a una aplicación móvil educativa, para incorporar la capacidad de identificar el estado afectivo del estudiante y realizar acciones orientadas a mejorar su aprendizaje. Como parte de la contribución del trabajo se generó una base de datos afectiva mediante EmoData, la aplicación móvil desarrollada. Nuestro trabajo es innovador pues está enfocado a detectar emociones relacionadas con el aprendizaje a través del uso natural de los dispositivos móviles, por lo cual su campo de aplicaciones es vasto.

El contenido del artículo se detalla en el siguiente orden: trabajos relacionados con el reconocimiento de emociones son presentados en la Sección 2; la implementación de la aplicación EmoData y su funcionamiento se encuentran en la Sección 3; el experimento para la creación de una base de datos afectiva se muestra en la Sección 4; la implementación del sistema de reconocimiento de emociones se presenta en la Sección 5 y finalmente en la Sección 6 se exponen las conclusiones y trabajo futuro.

\section{Trabajos relacionados}

En esta sección se presentan algunos importantes trabajos de investigación en el campo del reconocimiento de emociones en dispositivos móviles.

El sistema de reconocimiento móvil presentado por Wu et al. [10] capta imágenes del rostro y realiza el reconocimiento facial con base en distancias entre rasgos faciales y capta las señales de voz y las procesa con base a Coeficientes Cepstrales en las Frecuencias de Mel (MFCCs), Delta MFCC y Delta-Delta MFCC. Este sistema utiliza una máquina de soporte vectorial (SVM por sus siglas en inglés) para clasificar y reconocer las emociones: neutral, felicidad, enojo y tristeza. El porcentaje de efectividad promedio logrado es del $87 \%$.

MoodScope [11] es una aplicación móvil desarrollada para el sistema operativo iOS, que deduce el estado de ánimo del usuario con base al uso de su celular, basado en un modelo estadístico que utiliza información de los SMS, correos electrónicos, llamadas telefónicas, buscador Web y la ubicación del dispositivo para inferir el estado de ánimo del usuario. Esta aplicación es transparente para el usuario ya que se ejecuta en segundo plano, registra la interacción del usuario con su celular y almacena los datos en un servidor a través de una conexión de datos o Wi-Fi.

EmotionSense [12] es una aplicación móvil para detectar la emoción del usuario con base a los hábitos de uso del dispositivo. Esta aplicación registra la ubicación física donde se encuentra el dispositivo y como se está moviendo, el ruido del entorno y además monitorea las llamadas y mensajes, con el objetivo de desarrollar un patrón de hábitos del usuario que le permitan deducir su emoción. Los componentes que utiliza son: GPS, acelerómetro y micrófono.

Samurai fruit [13] es una variación de un juego para dispositivos móviles para iPhone que reconoce la emoción del jugador con base al comportamiento de sus gestos táctiles en la pantalla del dispositivo. Este juego consiste en estrujar y cortar frutas a través de movimientos táctiles; mientras el usuario está jugando, la aplicación captura y registra las coordenadas de cada punto de un golpe, el área de contacto del dedo a 
cada punto y la duración del tiempo de un golpe. El área de contacto se utiliza para medir la presión ejercida por los jugadores. Los estados emocionales que identificaron son: Emocionado, Relajado, Frustrado y Aburrido.

\section{Implementación de EmoData}

EmoData es una aplicación móvil desarrollada para el sistema operativo Android, esta se encarga de monitorear y recolectar información de los componentes del celular para relacionarlos con el estado afectivo y la postura corporal del usuario. Los componentes del celular con los que trabaja son micrófono, acelerómetro y giroscopio.

En la Figura 1 se puede observar la arquitectura de esta aplicación, la cual está formada por las capas de presentación, recolección y datos.

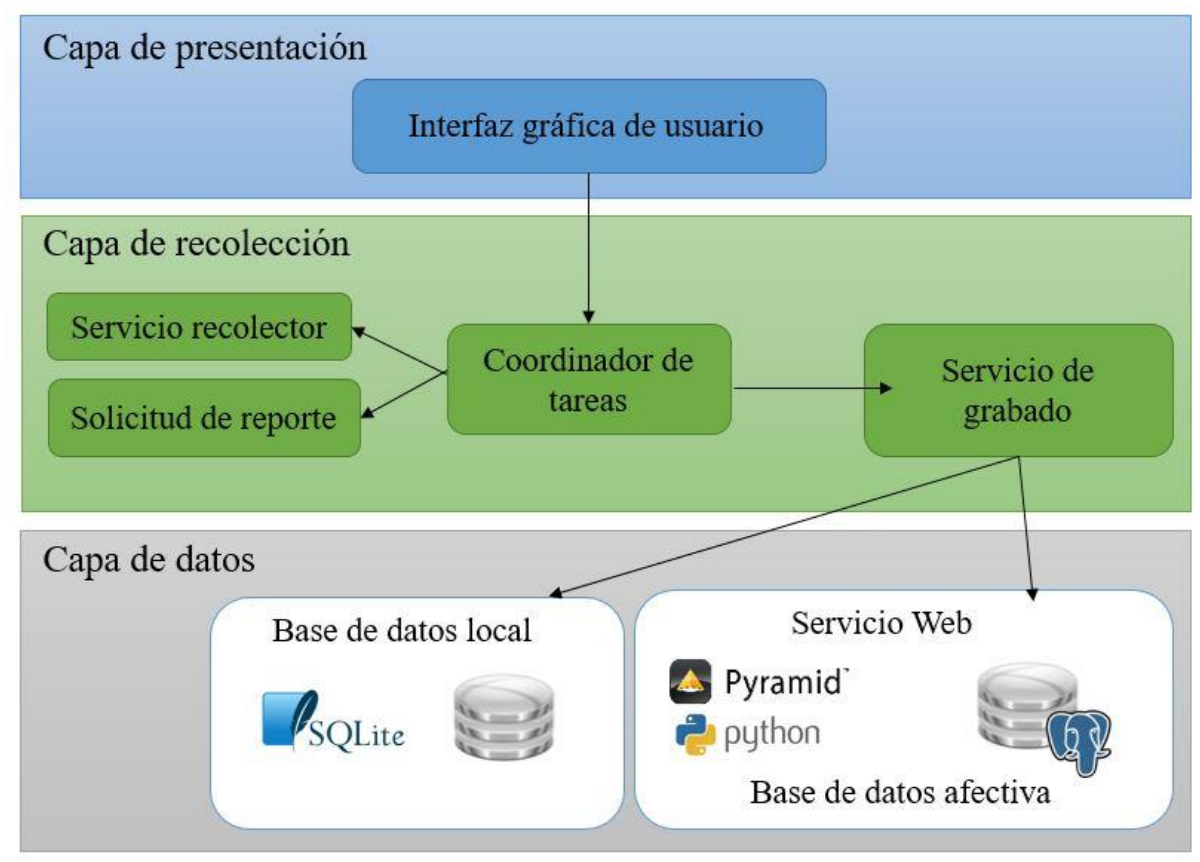

Fig. 1. Arquitectura de EmoData.

Capa de presentación: En esta capa se encuentran las interfaces de EmoData que permiten al usuario interactuar con el sistema para que pueda registrarse, identificarse e iniciar la tarea de recolección. Esta capa utiliza una API de Facebook cuando el usuario desea utilizar su cuenta de Facebook para identificarse.

Capa de recolección: Esta capa se encarga de monitorear y recolectar la información de los componentes del dispositivo y está formada por 2 módulos y 2 servicios explicados a continuación.

- Coordinador de tareas: Dirige las tareas principales de la aplicación. Solicita que el servicio recolector obtenga la información de los sensores y después continúa 
Sistema de reconocimiento multimodal de emociones relacionadas al aprendizaje en dispositivos ...

pidiendo al usuario que ingrese su emoción y postura, por último envía al servicio de grabado la información recolectada, la emoción y postura para que realice el almacenamiento de los datos.

- Servicio recolector: Este servicio se encarga de recolectar el audio recibido por medio del micrófono y la secuencia de valores de los ejes $\mathrm{X}, \mathrm{Y}$ y $\mathrm{Z}$ tanto del acelerómetro como del giroscopio. El audio del micrófono se recolecta en un archivo de audio con formato .pcm y la información del acelerómetro y giroscopio se recolecta como arreglos en formato JSON.

- Solicitud de reporte: Este módulo solicita al usuario que ingrese al sistema su estado emocional y la postura en que se encuentra, con el objetivo de etiquetar la información recolectada automáticamente.

- Servicio de grabado: Este servicio envía al servidor la información recolectada y etiquetada con la emoción y postura del usuario. Se utiliza la librería Volley que hace una solicitud asíncrona HTTP a un servicio Web desarrollado en Python y su Framework Pyramid. El archivo de audio se convierte a formato .wav y se serializa en una cadena base 64antes para facilitar su envío al servidor, pero la información se almacena localmente en la base de datos SQLite, si no se cuenta con conexión a Internet.

Capa de datos: Esta capa contiene la base de datos afectiva donde se almacena la información, una parte se encuentra en el dispositivo y otra en el servidor.

\subsection{Funcionamiento de la aplicación EmoData}

En la Figura 2 se esquematiza el funcionamiento de la aplicación EmoData utilizada para la recolección de las señales del dispositivo que se usan para determinar la emoción del usuario.

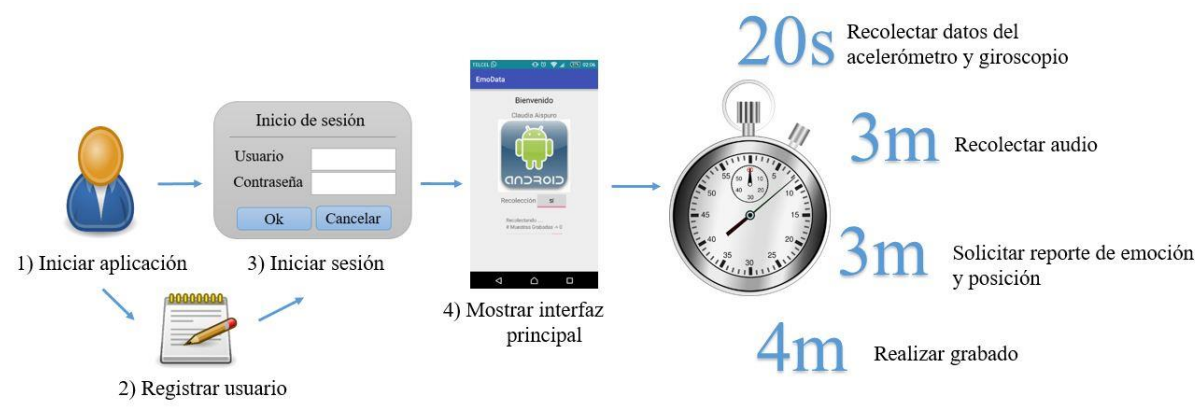

Fig. 2. Funcionamiento de EmoData.

A continuación se detalla el funcionamiento de EmoData.

1. El usuario inicia la aplicación y el sistema presenta la interfaz inicial del sistema (ver a) Figura 3), donde el usuario puede registrarse o iniciar sesión.

2. Un usuario puede registrarse para iniciar sesión proporcionando sus datos (nombre, número de celular y correo electrónico) y posteriormente ingresa con su usuario y contraseña. 
3. El usuario puede iniciar sesión por medio de Facebook o con su usuario y contraseña. Una vez que inicie sesión, la aplicación presenta su interfaz principal mostrada en b) Figura 3.

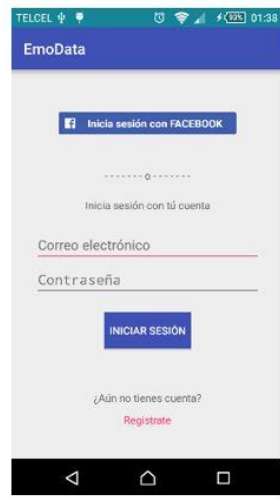

a)

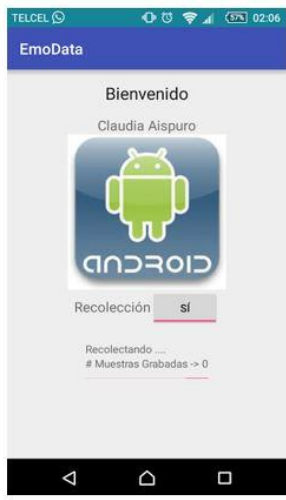

b)

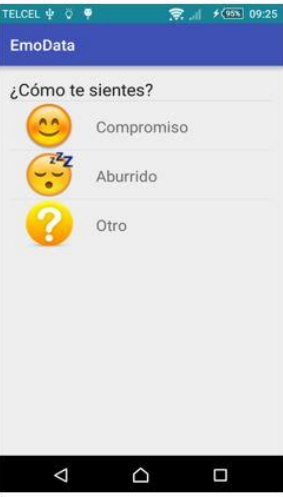

c)

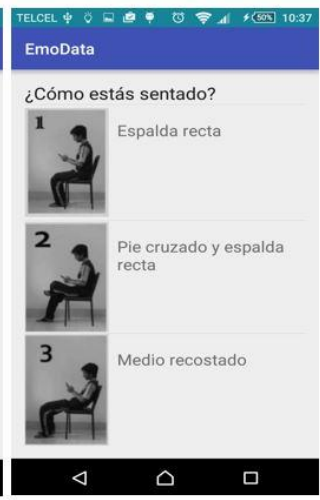

d)

Fig. 3. Interfaces de EmoData. Las imágenes de la pantalla donde aparecen las posiciones del estudiante fueron tomadas en el artículo de Hosain et al[14].

4. En la interfaz principal, el usuario indica si la tarea de recolección estará activada o no. Durante el proceso de recolección, la aplicación ejecuta tareas recurrentes:

- Cada 20 segundos se recolectan los valores de los ejes del acelerómetro y giroscopio. El acelerómetro es un dispositivo que detecta las fuerzas de la aceleración y puede medir la aceleración en uno, dos o tres ejes, siendo los más comunes los de tres ejes. Entre los usos del acelerómetro se encuentran detectar el movimiento e inclinación. Por otra parte, el giroscopio es un sensor que permite medir las velocidades angulares de tres ejes, mantener y cambiar la orientación del dispositivo. Un ejemplo de las aplicaciones de estos sensores, es el trabajo de Paredes et al [15] en el cuál se utilizan un acelerómetro y giroscopio para modelar las oscilaciones del brazo durante el uso común del celular. Otra aplicación [16] es utilizar estos sensores para diferencias posturas estáticas (estar de pie, flexionado, sentado o acostado) de posturas dinámicas.

- Para recolectar la información del acelerómetro y giroscopio la aplicación móvil se comunica con el sistema de sensores y a partir de una instancia de la clase Sensor Manager obtiene acceso a estos sensores para captar los valores de los ejes $\mathrm{X}, \mathrm{Y}$ y Z en ese momento.

- El audio recibido por el micrófono se recolecta y almacena en archivos con una duración de 3 minutos por periodo.

- Cada tres minutos, la aplicación solicita al usuario que reporte su estado afectivo y postura por medio de las interfaces mostradas en c) y d) Figura 3.

- El almacenamiento de la información se realiza cada cuatro minutos y como se observa en la Figura 4 consiste en enviar la información a un servidor Web en caso de contar con conexión a Internet, si no es posible, entonces se 
Sistema de reconocimiento multimodal de emociones relacionadas al aprendizaje en dispositivos ...

graba localmente la información para posteriormente volver a intentar enviarla.

- Cuando el usuario decide detener la recolección, la aplicación detiene todas las tareas que esté ejecutando.

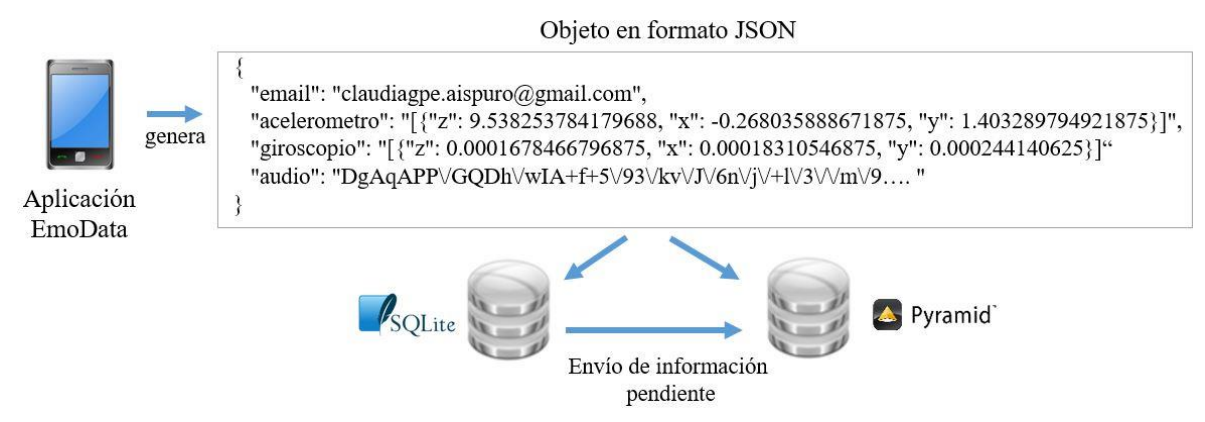

Fig. 4. Grabado de la aplicación EmoData.

\section{Método del experimento para la creación de la base de datos afectiva}

El objetivo de este experimento es crear una base de datos afectiva de emociones espontáneas que almacene un audio y secuencias de valores de los ejes del acelerómetro y giroscopio etiquetados con la emoción y postura corporal reportadas por el usuario.

El experimento consistió en utilizar la aplicación educativa Duolingo[17] y EmoData para generar la información que se almacenará en la base de datos. Los sujetos de prueba fueron veintiséis estudiantes del Instituto Tecnológico de Culiacán, de los cuales veintiuno son hombres y cinco mujeres de diferentes edades, que van de 18 a 30 años.

La base de datos afectiva fue generada a través de una secuencia de pasos, que se describen a continuación.

Estímulo: Para provocar las emociones se utilizó la aplicación educativa DuoLingo, en la que se realizaron sesiones de práctica de tres minutos. DuoLingo presenta diferentes tipos de ejercicios que pueden ser traducciones de inglés a español y viceversa, ejercicios de pronunciación, escribir la frase que se escucha, ejercicios de opción múltiple, entre otros.

Recolección de señales: Mientras el estudiante practicaba con DuoLingo, las señales de audio e información de los sensores acelerómetro y giroscopio eran registradas por medio de la aplicación EmoData.

Extracción de características: Se desarrolló una aplicación en Java que utiliza la herramienta MusicG[18] para extraer las características de las grabaciones de audio: tono e intensidad y se implementaron los métodos de extracción de características media, desviación estándar de los valores de cada uno de los ejes del acelerómetro y giroscopio y el coeficiente de correlación de los ejes XY.

Clasificación: La clasificación fue realizada automáticamente considerando la posición y emoción reportada por el estudiante. 
La base de datos afectiva por el momento está compuesta por 85 registros (entre 3 y 4 por cada estudiante) clasificados con su respectiva emoción y postura corporal.

\section{Implementación del sistema de reconocimiento de emociones}

El sistema reconocedor de emociones recibe las señales afectivas del usuario y las analiza para retornar como resultado la emoción del usuario. En esta sección se presenta la arquitectura Cliente-Servidor del sistema de reconocimiento. Esta arquitectura se muestra en la Figura 5.

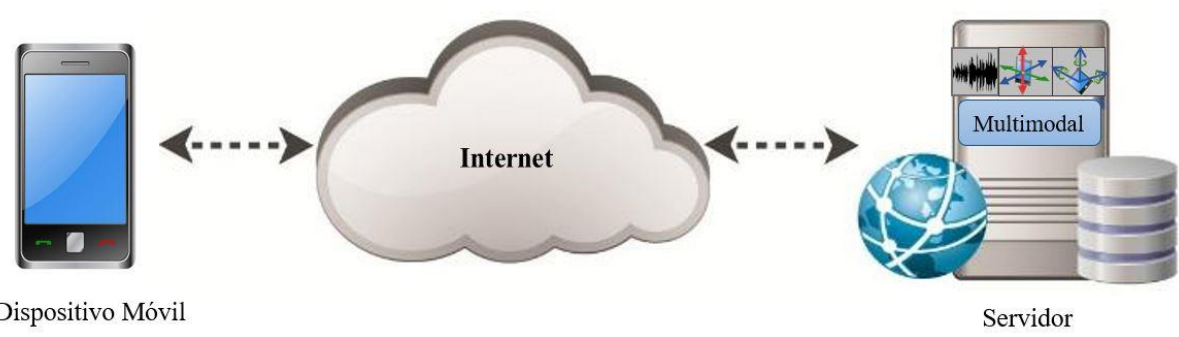

Fig. 5. Arquitectura del Sistema Reconocedor.

El Cliente, en este caso un dispositivo móvil realiza las peticiones o solicitudes de reconocimiento al Servidor, para que realice la tarea de reconocimiento. La arquitectura del servidor está formada por las capas: Servicios Web, Reconocimiento y Datos.

Capa de Servicios Web. La capa de servicios Web está implementada en un servicio Web encargado de recibir las solicitudes de los usuarios y responder con la emoción identificada. En su solicitud, el usuario especifica si desea que se realice reconocimiento unimodal o multimodal y proporciona las señales afectivas para realizar el reconocimiento. Si el reconocimiento es de audio, la señal afectiva es el audio serializado, con la finalidad de facilitar su envío. En el caso del reconocimiento por medio de la postura del usuario, la señal afectiva es la secuencia de valores de los ejes del acelerómetro y por último, en el reconocimiento por medio del movimiento, se utilizan dos señales afectivas, una es la secuencia de valores de los ejes del acelerómetro y la otra una secuencia de valores de los ejes del giroscopio.

Capa de Reconocimiento. La capa de Reconocimiento esta implementada en una librería Java encargada de realizar el proceso de reconocimiento de la emoción con la información que provee la capa de servicio web. Esta librería permite realizar el reconocimiento de forma unimodal, que puede ser por voz, postura o movimiento del usuario o multimodal usando una combinación de los anteriores.

Reconocimiento por voz. Este proceso mostrado en la Figura 6, recibe como entrada un audio en formato wav serializado en Base64 e inicia con la etapa de preprocesamiento, en donde el audio es deserializado y convertido en una onda de audio normalizada a la cual se le extraen dos características: tono e intensidad. La extracción de características se realiza mediante la herramienta MusicG, por medio de esta herramienta se obtienen 4 valores de las dos características: el valor máximo y mínimo del tono y el valor máximo y mínimo de intensidad. Estas características conforman el 
vector de características que sirve como entrada a una SVM incluida en la librería LibSVM [19], para obtener la emoción. La máquina de soporte vectorial fue entrenada previamente con la información de la base de datos afectiva.

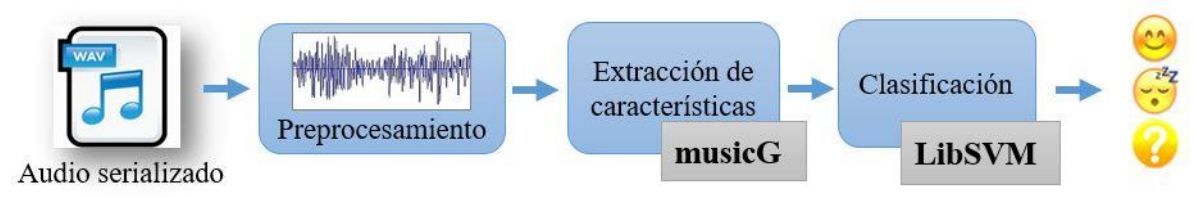

Fig. 6. Algoritmo reconocedor de voz.

Reconocimiento por medio de la posición. Este reconocimiento está basado en el trabajo de Hossain et al. [14], y se muestra en la Figura 7. La entrada es la secuencia de valores de cada uno de los ejes del acelerómetro y en la etapa de preprocesamiento se formatean los datos para facilitar la extracción de sus características.

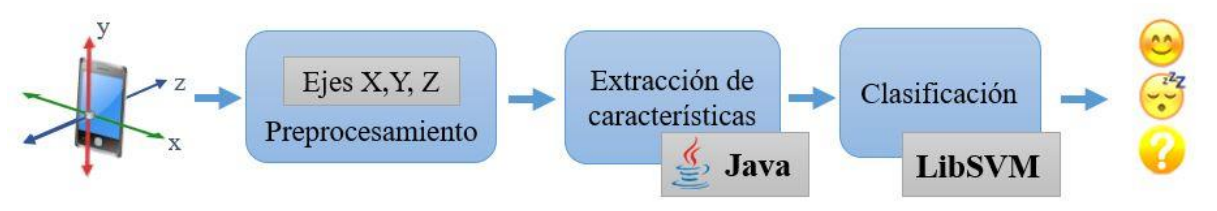

Fig. 7. Reconocimiento por posición.

Para la extracción, se revisaron las técnicas de procesamiento sobre el reconocimiento del contexto a partir de datos del acelerómetro [20] y se seleccionaron algunas características del dominio de tiempo (técnicas matemáticas y estadísticas) que con frecuencia son usadas para extraer información básica de los datos crudos del acelerómetro en algoritmos de reconocimiento de actividad. Entre las características que están disponibles en el dominio de tiempo se encuentran: media, mediana, varianza, desviación estándar, mínimo, máximo, rango, media cuadrática RMS, coeficiente de correlación de señales, entre otros. La media es un promedio de un conjunto de valores o su distribución. Muchas investigaciones han utilizado la media para identificar la postura del usuario (sentado, de pie parado, etc.) [21] y también para diferenciar si la actividad es estática y dinámica [22], ente otros usos.

La varianza es la media de las diferencias con la media elevadas al cuadrado. Y la desviación estándar es la raíz cuadrada de la varianza y representa la variabilidad de un conjunto de datos y la probabilidad de distribución. Los coeficientes de correlación determinan el grado de correlación entre las señales de varios ejes y sirven para medir la fuerza y dirección de una relación lineal entre dos señales. Uno de los más utilizados es el coeficiente de correlación de Pearson. Una de las aplicaciones de la correlación de señales es para diferenciar entre actividades que involucran movimiento en una sola dimensión [23].

Este sistema de reconocimiento extrae la media y la desviación estándar para cada uno de los ejes del acelerómetro y el coeficiente de correlación de Pearson de los ejes XY. 
El vector de características está formado por 7 valores: la media y desviación estándar de cada uno de los ejes forman 6 valores y el coeficiente de correlación de Pearson de los ejes XY es el último. Este vector fue creado con un método implementado en Java que extrae las características de las señales. En base a estas características se realiza la clasificación utilizando la SVM de la librería LibSVM previamente entrenada con la información de la base de datos afectiva.

Reconocimiento por medio de movimiento. Este proceso de reconocimiento es llamado "por movimiento" debido a que trabaja con la información de dos sensores de movimiento, tal como se muestra en la Figura 8. Recibe como entrada dos secuencias de valores de los ejes del acelerómetro y del giroscopio. En la etapa de preprocesamiento se formatean los datos para facilitar la extracción de las características. Las características que se extraen son la media y desviación estándar de cada uno de los ejes, por lo que el vector de características está formado por estos 12 valores. Este proceso se realiza con un método implementado en Java. En base a estas características se realiza la clasificación utilizando la máquina de soporte vectorial SVM de la librería LibSVM previamente entrenada con la información de la base de datos afectiva.
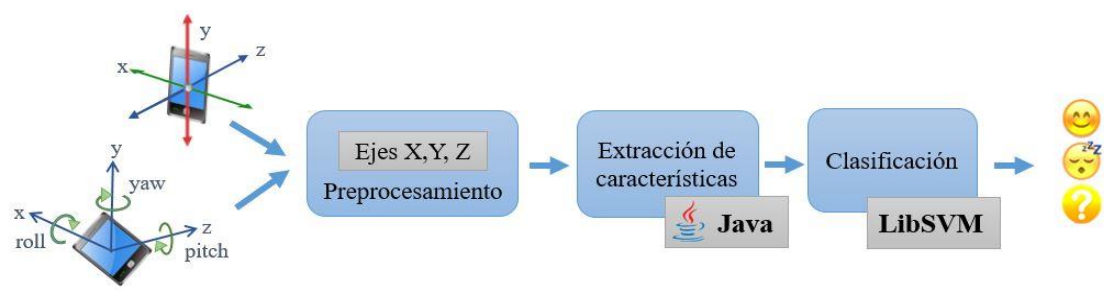

Fig. 8. Reconocimiento por movimiento.

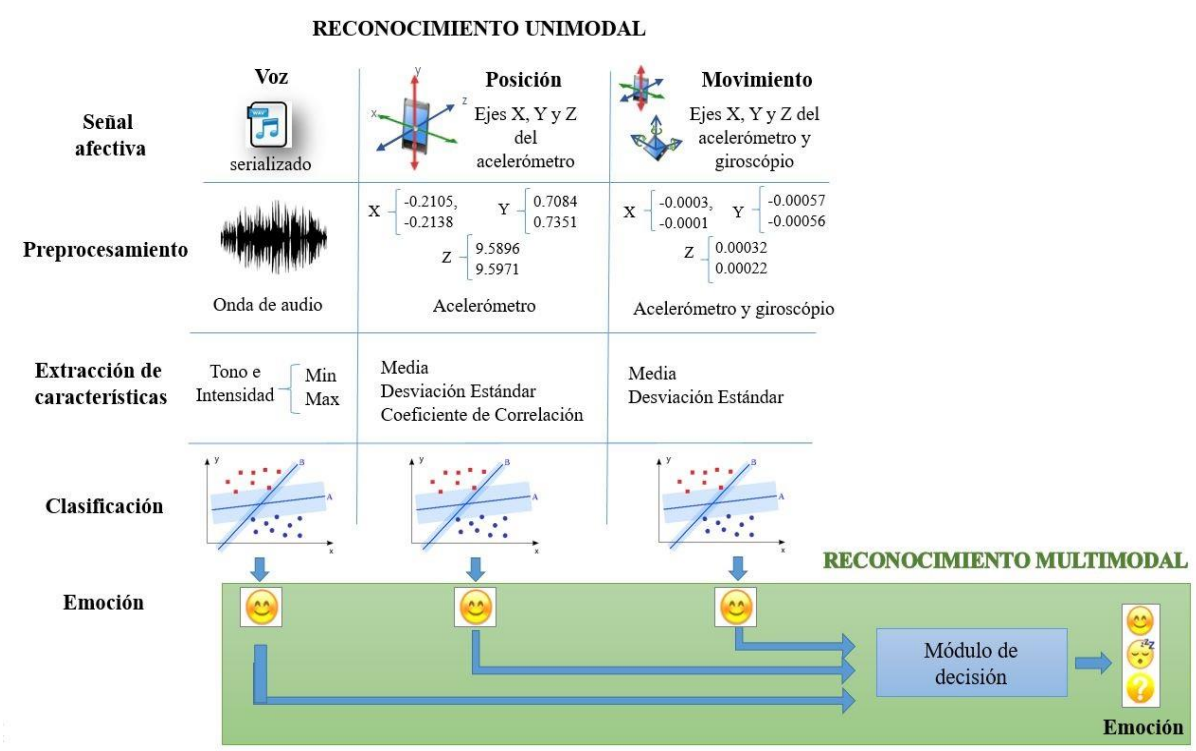

Fig. 9. Proceso de Reconocimiento Multimodal. 
Reconocimiento multimodal. Este proceso se representa de forma general en la Figura 9, recibe como entrada las tres señales afectivas: el audio, los datos del acelerómetro y los datos del giroscopio. El método de fusión que se utiliza para combinar las modalidades es a nivel decisión, esto significa que primero se realiza el reconocimiento de emoción de cada una de forma unimodal, identificándose tres emociones, una por cada tipo de reconocimiento realizado y después se fusionan para obtener la emoción final.

El módulo de decisión se encarga de realizar la fusión, este módulo recibe las tres emociones identificadas en los reconocedores unimodales que sirven como entrada a un motor de inferencias difusas por medio de la librería jFuzzyLogic[24] que ejecuta un conjunto de reglas difusas, para determinar la emoción final que puede ser Compromiso, Aburrimiento u Otro, este último significa que la emoción no pudo ser clasificada en las otras dos emociones. Un ejemplo de regla difusa se puede observar en la Figura 10.

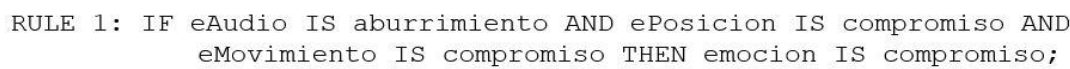

Fig. 10. Ejemplo de una regla difusa.

Capa de datos. Esta capa se encuentra la base de datos afectiva utilizada para realizar el entrenamiento de la máquina de soporte vectorial SVM.

\section{Conclusiones y trabajos futuros}

El sistema desarrollado es capaz de realizar el reconocimiento de emociones utilizando la información generada por los sensores de un dispositivo móvil.

El sistema de reconocimiento está implementado de tal manera que puede ser utilizado por diversos tipos de aplicaciones móviles entre ellas, aplicaciones educativas como tutores, juegos o cualquier otra aplicación que necesite incorporar el reconocimiento de emociones de forma continua, no invasiva y en el ambiente natural para el usuario.

El sistema reconocedor está disponible para su uso a través de un servicio web, el cual recibe los datos de entrada (el audio en formato wav serializado, la cadena en formato JSON con la secuencia de los valores de los ejes del acelerómetro y giroscopio) y retorna la emoción identificada como salida. Un ejemplo de la información que el servicio web espera recibir se muestra en la Figura 11.

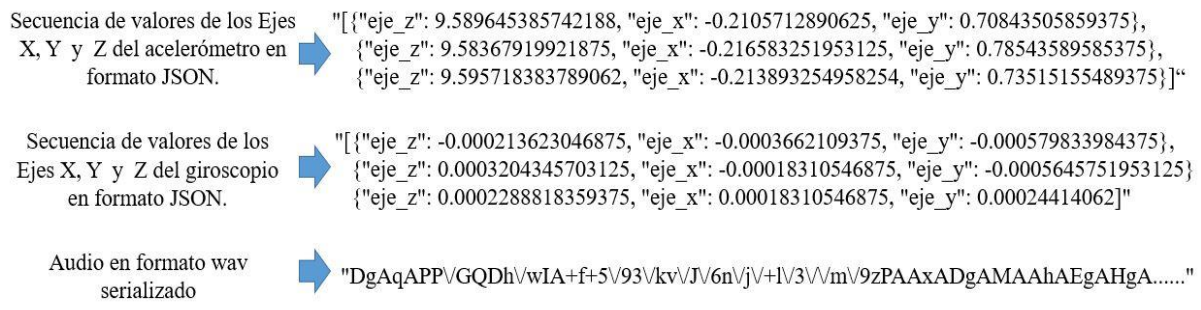

Fig. 11. Ejemplo de los datos de entrada del servicio Web. 
Como trabajo futuro se espera realizar nuevas pruebas de sistema de reconocimiento de emociones para determinar la precisión del reconocimiento logrado y analizar los resultados. Así como mejorar el reconocimiento por voz e implementar otras formas de reconocimiento emocional basados en los sensores del celular y agregar la identificación de más emociones relacionadas al aprendizaje.

También se pretende experimentar utilizando otra aplicación educativa para crear la base de datos afectiva y ver cómo influye en la tasa de reconocimiento del sistema.

\section{Referencias}

1. Calvo, R.A., D’Mello, S.: Affect detection: An interdisciplinary review of models, methods, and their applications. IEEE Trans. Affect. Comput., Vol. 1, No. 1, pp. 18-37, (2010)

2. Kory, K.D., Sidney, J.: A Review and Meta-Analysis of Multimodal Affect Detection. Vol. 20, No. 10 (2015)

3. Zhang, S., Hui, P.: A Survey on Mobile Affective Computing. No. 1 (2014)

4. Langner, O., Dotsch, R., Bijlstra, G., Wigboldus, D.H.J., Hawk, S.T., van Knippenberg, A.: Presentation and validation of the Radboud Faces Database. Cogn. Emot., Vol. 24, No. 8, pp. 1377-1388 (2010)

5. Baker, R., Rodrigo, M., Xolocotzin, U.: The dynamics of affective transitions in simulation problem-solving environments. 2nd International Conference on Affective Computing and Intellegent Interaction, pp. 666-677 (2007)

6. D'Mello, S.: A selective meta-analysis on the relative incidence of discrete affective states during learning with technology. J. Educ. Psychol., Vol. 105, No. 4, pp. 1082-1099 (2013)

7. D'Mello, S.K., Graesser, A.: The half-life of cognitive-affective states during complex learning. Cogn. Emot., Vol. 25, No. 7, pp. 1299-308 (2011)

8. Baker, R.S.J.D., D’Mello, S.K., Rodrigo, M.M.T., Graesser, A.C.: Better to be frustrated than bored: The incidence, persistence, and impact of learners' cognitive-affective states during interactions with three different computer-based learning environments. Int. J. Hum. Comput. Stud., Vol. 68, No. 4, pp. 223-241 (2010)

9. PuroMarketing, http://www.puromarketing.com/96/26113/uso-apps-moviles-aumentodurante.html.

10. Wu, Y., Lin, S., Yang, D.: A Mobile Emotion Recognition System Based on Speech Signals and Facial Images. pp. 212-217 (2013)

11. Likamwa, R., Liu, Y., Lane, N.D., Zhong, L.: MoodScope: Building a Mood Sensor from Smartphone Usage Patterns. MobiSys '13 Proceeding 11th Annu. Int. Conf. Mob. Syst. Appl. Serv., pp. 389-402 (2013)

12. Rachuri, K.K., Musolesi, M., Mascolo, C., Rentfrow, P.J., Longworth, C., Aucinas, A.: EmotionSense: A Mobile Phones based Adaptive Platform for Experimental Social Psychology Research. Int. Stud., pp. 281-290 (2010)

13. Gao, Y., Bianchi-Berthouze, N., Meng, H.: What Does Touch Tell Us about Emotions in Touchscreen-Based Gameplay? ACM Trans. Comput. Interact., Vol. 19, No. 4, pp. 1-30, (2012)

14. Bin Hossain, R., Sadat, M., Mahmud, H.: Recognition of human affection in smartphone perspective based on accelerometer and user's sitting position. 17th International Conference on Computer and Information Technology, ICCIT, pp. 87-91 (2015)

15. Paredes, P., Berkeley, U.C., Sun, D.: Sensor-less Sensing for Affective Computing and Stress Management Technology. Pervasive Computing Technologies for Healthcare and Workshops, Sensor-less Sensing for Affective Computing and Stress Management Technology, pp. 459-463 (2013) 
Sistema de reconocimiento multimodal de emociones relacionadas al aprendizaje en dispositivos ...

16. Li, Q., Stankovic, J.A., Hanson, M.A., Barth, A.T., Lach, J., Zhou, G.: Accurate, fast fall detection using gyroscopes and accelerometer-derived posture information. Proceedings 6th International Workshop on Wearable and Implantable Body Sensor Networks, BSN, pp. 138-143 (2009)

17. Duolingo: Https://es.duolingo.com/

18. MusicG: Https://code.google.com/archive/p/musicg/

19. Chang, C.-C., Lin, C.-J.: LIBSVM: A Library for Support Vector Machines. ACM Trans. Intell. Syst. Technol., Vol. 2, No. 3 (2011)

20. Figo, D., Diniz, P.C., Ferreira, D.R., Cardoso, J.M.P.: Preprocessing techniques for context recognition from accelerometer data. Pers. Ubiquitous Comput., Vol. 14, No. 7, pp. 645$662(2010)$

21. Jeong, D.U., Kim, S.J., Chung, W.Y.: Classification of posture and movement using a 3axis accelerometer. International Conference on Convergence Information Technology, ICCIT, pp. 837-844 (2007)

22. Kawahara, Y., Kurasawa, H., Morikawa, H.: Recognizing User Context Using Mobile Handsets with Acceleration Sensors. IEEE International Conference on Portable Information Devices, pp. 1-5 (2007)

23. Ravi, N., Dandekar, N., Mysore, P., Littman, M.: Activity recognition from accelerometer data. Proc. Natl., pp. 1541-1546 (2005)

24. Cingolani, P., Alcalá-Fdez, J.: JFuzzyLogic: A robust and flexible Fuzzy-Logic inference system language implementation. IEEE International Conference on Fuzzy Systems (2012) 